\title{
Pump fluence dependence of ultrafast carrier dynamics in bulk $\mathrm{ZnSe}$
}

\author{
Gaofang Li, Fenghong Chu, Anduo Hu, Liang Xue, \\ Zhenglan Bian, Chengxin Pang \\ Shanghai University of Electric Power, \\ Shanghai 200090, P. R. China \\ E-mail:li_gaofang@163.com
}

\author{
Hong Ma \\ Shandong provincial key laboratory of optics and photonic \\ device, College of Physics and Electronics, Shandong \\ Normal University, Jinan 250014, China
}

\begin{abstract}
Ultrafast carrier dynamics of bulk $\mathrm{ZnSe}$ is investigated by means of the optical-pump terahertz-probe spectroscopy with pump fluence ranging from 38 to $239 \mu \mathrm{J} / \mathrm{cm}^{2}$ at room temperature. With the laser pulse excitation at $400 \mathrm{~nm}$, the negative transmission of terahertz pulse shows an ultrafast rising followed by a biexponential recovery. The relaxation time of fast decay and slow one is increased with pump fluence. This trend is expected for surface states filling, which may inhibit carrier lifetime.
\end{abstract}

Keywords-bulk ZnSe; optical-pump terahertz-probe spectroscopy; carrier dynamics

\section{INTRODUCTION}

Zinc selenide ( $\mathrm{ZnSe}$ ), with a room temperature band gap of $2.7 \mathrm{ev}$ [1], possesses unique optical and photovoltaic properties and exhibits great attractive applications, such as blue-green light emitting diodes, photo-luminescent and electroluminescent devices, lasers, thin film solar cell, nonlinear optical crystal and infrared optical material [2, 3]. Optical pump terahertz-probe (OPTP) technology is recently developed and is proven to be a powerful tool to study the transient carrier dynamics in photoexcited materials in subpicosecond time scale [4]. Much work has been done by using OPTP techniques to investigate nonequilibrium dynamics in photoexcited materials [5-10], but little is done in bulk $\mathrm{ZnSe}$.

In this study, the ultrafast carrier dynamics of bulk $\mathrm{ZnSe}$ are investigated by means of the OPTP technique with pump fluence ranging from 38 to $239 \mu \mathrm{J} / \mathrm{cm}^{2}$ at room temperature. With the laser pulse excitation at $400 \mathrm{~nm}$, the negative transmission of terahertz pulse shows an ultrafast rising followed by a biexponential recovery. The relaxation time of fast decay and slow one is increased with pump fluence. This trend is expected for surface states filling, which may inhibit carrier lifetime.

\section{EXPERIMENTAL ARRANGEMENTS}

The experimental setup for OPTP is shown in Fig. 1. The source of optical pulses in the experiment comes from a Ti: sapphire regenerative amplifier (Spectra-Physics, Spitfire Pro.) with pulse duration of $120 \mathrm{fs}$ at a center wavelength of $800 \mathrm{~nm}$ and repetition rate of $1 \mathrm{kHz}$. The output beam is split into three portions, which correspond to $\mathrm{THz}$ generation, probe, and pump beams. The THz pulse is generated by slightly focusing $40 \mathrm{~mW}$ of the generation beam on a $1 \mathrm{~mm}$-thickness-ZnTe crystal with (110)-orientation. The $\mathrm{THz}$ radiation is detected by free-space electro-optic sampling in a 1-mm-thick (110)orientated ZnTe crystal with the weak probe pulse. The signal of $\mathrm{THz}$ is sampled with a balanced photodetector connected with a lock-in amplifier. The $\mathrm{THz}$ radiation was focused onto the sample with a spot diameter of $1.5 \mathrm{~mm}$. The pump beam is frequency-doubled to a violet beam at $400 \mathrm{~nm}$ by a $1 \mathrm{~mm}$-long BBO crystal. The sample was photo-excited with a pump pulse at wavelength of $400 \mathrm{~nm}$. The spot diameter of pump beam is about $4 \mathrm{~mm}$, which is two times larger than that of the $\mathrm{THz}$ beam to ensure uniform pump-beam illumination. The average pump fluence varying from 38 to $239 \mu \mathrm{J} / \mathrm{cm}^{2}$ was obtained via an adjustable optical attenuator. The sample studied in this work is bulk ZnSe with thickness of $0.5 \mathrm{~mm}$, orientation of (0001). The entire OPTP setup is enclosed in a chamber purged with dry nitrogen to reduce water vapor absorption. All the experiments were carried out at room temperature.

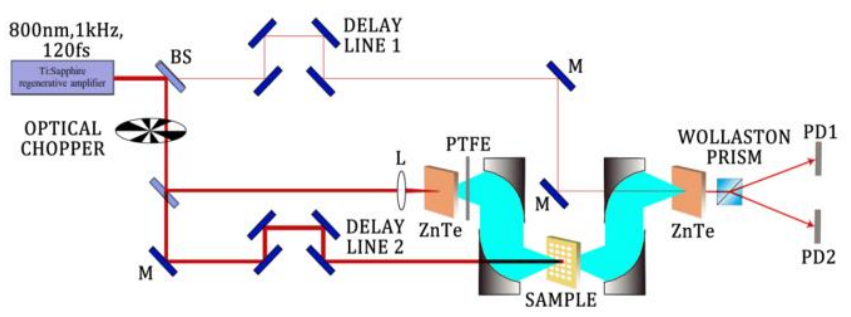

Fig. 1. Experimental arrangement for optical pump-terahertz probe measurements.

\section{RESULT AND DISCUSSIONS}

Figure 2 shows the UV-visible absorption spectra in the region of $350-800 \mathrm{~nm}$ for bulk $\mathrm{ZnSe}$ at room temperature. It is seen that the absorption at $400 \mathrm{~nm}(3.1 \mathrm{eV})$ is large enough for bulk $\mathrm{ZnSe}$, which indicates that 400-nm-pumping can effectively induce carrier redistribution in the conduction band.

The dynamics of photoexcited electrons were measured by time-resolved THz-TDS using an OPTP setup. Fig. 3 shows the negative pump-induced change (normalized) in the peak of the $\mathrm{THz}$ electric field pulse transmitted, $-\Delta \mathrm{T} / \mathrm{T}_{0}$, for different 


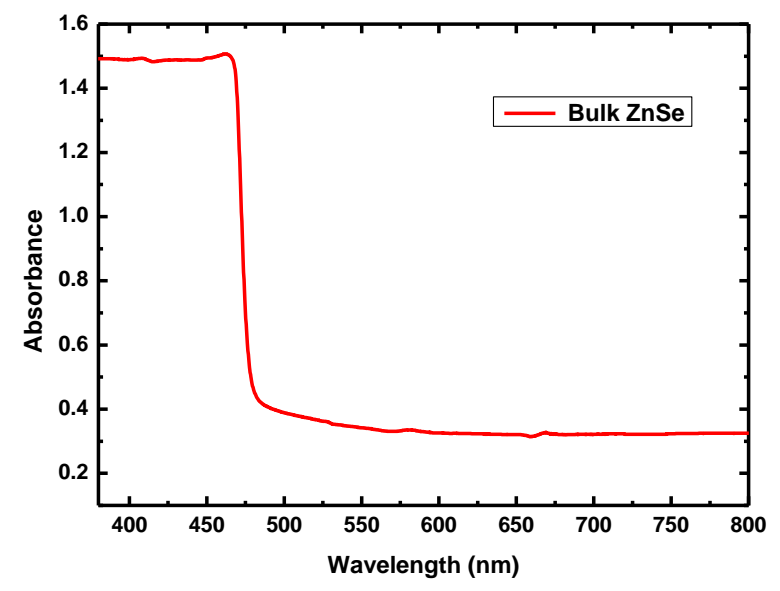

Fig. 2. The absorbance spectrum of $\mathrm{ZnSe}$ nanocrystal at room temperature.

pump-probe delays t, through the bulk $\mathrm{ZnSe}$ with the excitation fluence of 38,71 and $239 \mu \mathrm{J} / \mathrm{cm}^{2}$ at $400 \mathrm{~nm}$, where $\Delta \mathrm{T}=\mathrm{T}-\mathrm{T}_{0}$, $\mathrm{T}$ and $\mathrm{T}_{0}$ are the transmitted intensity of $\mathrm{THz}$ peak through the sample with and without excitation, respectively. The negative transmitted $\mathrm{THz}$ pulses through bulk ZnSe instantaneously rise upon the arrival of the pump pulses. It can be seen that the carrier lifetime is increased with pump fluence. The transient negative differential transmission of bulk $\mathrm{ZnSe}$ for different pump fluence, as shown in Fig. 3, can be well fitted with a biexponential function (see solid curves), and the fitting parameters are presented in Table 1. It can be seen that the relaxation time of fast decay and slow one is increased with pump fluence. This trend is expected for surface states filling [11], which may inhibit carrier lifetime.

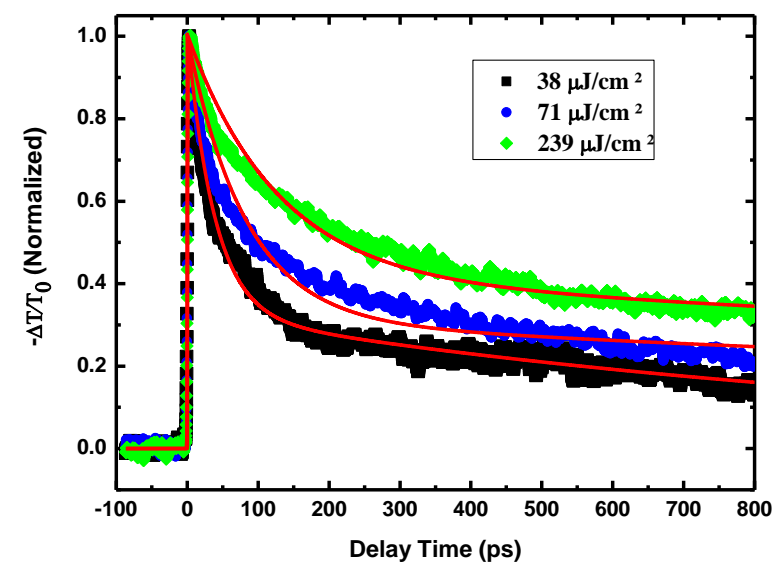

Fig. 3. The negative differential transmission $\left(-\Delta \mathrm{T} / \mathrm{T}_{0}\right)$ (normalized) of the main peak of the $\mathrm{THz}$ probe pulse as a function of time delay with pump fluence of 38,71 and $239 \mu \mathrm{J} / \mathrm{cm}^{2}$ for bulk $\mathrm{ZnSe}$. Solid curves are biexponential fits.

\begin{tabular}{ccc}
\hline $\begin{array}{c}\text { Pump fluence } \\
\left(\mu \mathrm{J} / \mathrm{cm}^{2}\right)\end{array}$ & $\tau_{1}(\mathrm{ps})$ & $\tau_{2}(\mathrm{ps})$ \\
\hline 38 & 38 & 1119 \\
71 & 81 & 3434 \\
239 & 125 & 4146 \\
\hline
\end{tabular}

Table 1. The fitting parameters with a biexponential function for pump fluence of 38,71 and $239 \mu \mathrm{J} / \mathrm{cm}^{2}$ with excitation at $400 \mathrm{~nm}$.

\section{CONCLUSIONS}

In summary, by using OPTP technique, the ultrafast carrier dynamics in bulk ZnSe with different pump fluence are investigated at room temperature. With the laser pulse excitation at $400 \mathrm{~nm}$, the negative transmission of terahertz pulse shows an ultrafast rising followed by a biexponential recovery. The relaxation time of fast decay and slow one is increased with pump fluence. This trend is expected for surface states filling, which may inhibit carrier lifetime.

\section{ACKNOWLEDGMENT}

This research is supported by National Natural Science Foundation of China (11304186 and 61205081), Shanghai University of Electric Power Scientific Research Fund (K2014028, K2014048, K2014018, K2014052 and K2014029), Opening Project from Key Laboratory of Specialty Fiber Optics and Optical Access Networks of Shanghai University, and Excellent Young Scholars Research Fund of Shandong Normal University.

\section{REFERENCES}

[1] K. D. Patel, G. K. Solanki, J. R. Gandhi, and S. G. Patel, "Structural and optical characterization of ZnSe crystals grown by physical vapor transport technique," Chalcogenide Letters, Vol. 6, No. 1, pp. 45-50, 2009.

[2] Wallace C H Choy, Sha Xiong and Yuxiu Sun, "A facile synthesis of zinc blende ZnSe nanocrystals", J. Phys. D: Appl. Phys. Vol. 42, pp. 125410, 2009.

[3] R. Bhargava (Ed.), Properties of wide bandgap II -VI, Semiconductors Inspection, London, 1997.

[4] D. G. Cooke, A. N. MacDonald, A. Hryciw, J. Wang, Q. Li, A. Meldrum, F. A. Hegmann, "Transient terahertz conductivity in photoexcited silicon nanocrystal films," Phys. Rev. B, Vol. 73, pp. 193311, 2006.

[5] M. Nakajima, N. Takubo, Z. Hiroi, Y. Ueda, and T. Suemoto, "Photoinduced metallic state in $\mathrm{VO}_{2}$ proved by the terahertz pump-probe spectroscopy," Appl. Phys. Lett., Vol. 92, pp. 011907, 2008.

[6] Y. Shi, Q. Zhou, C. Zhang, and B. Jin, "Ultrafast high-field carrier transport in GaAs measured by femtosecond pump-terahertz probe spectroscopy," Appl. Phys. Lett., Vol. 93, pp. 12115, 2008.

[7] M. Schall and P. Uhd Jepsen, "Above-band gap two-photon absorption and its inßuence on ultrafast carrier dynamics in ZnTe and CdTe," Appl. Phys. Lett., Vol. 80, No 25, pp. 4771-4773, 2002.

[8] M. Li, B. Wu, S.A. Ekahana, MlB Utama, G. Xing, Q. Xiong, T.C. Sum, $\mathrm{X}$. Zhang, "Size and surface effects on transient photoconductivity in 
CdS nanobelts probed by time-resolved terahertz spectroscopy," Appl. Phys. Lett., Vol. 101, pp. 091104, 2012.

[9] J. L. Hughes, E. C. Camus, M. D. Fraser, C. Jagadish, and M. B. Johnston, "Carrier dynamics in ion-implanted GaAs studied by simulation and observation of terahertz emission," Phys. Rev. B, Vol. 70 pp. 235330, 2004

[10] O. Ostroverkhova, D. G. Cooke, S. Shcherbyna, R. F. Egerton, F. A. Hegmann, R. R. Tykwinski, and J. E. Anthony, "Bandlike transport in pentacene and functionalized pentacene thin films revealed by subpicosecond transient photoconductivity measurements," Phys. Rev. B, Vol. 71, pp. 035204, 2005.

[11] T. S. Sosnowski, T. B. Norris, H. H. Wang, P. Grenier, J. F. Whitaker, and C. Y. Sung, "High-carrier-density electron dynamics in lowtemperature-grown GaAs" Appl. Phys. Lett., Vol. 70, pp. 3245, 1997. 